\title{
ShonA-Kunst im Palmengarten
}

Herbert Billensteiner

Bei der Shona-Bildhauerkunst handelt es sich um zeitgenössische Steinskulpturen aus dem südafrikanischen Land Simbabwe. Die meisten Künstlerinnen und Künstler gehören den Shona, der größten Volksgruppe Simbabwes, an. Darauf bezieht sich der Begriff SHonAKunst, der sich seit der Mitte des 20. Jh. etabliert hat. Die Shona umfassen neben vielen kleinen Stämmen die sechs Hauptvölker Kore Kore, Zezuru, Karanga, Manyika, Ndau und Rozvi. Im 19. Jh. hatte die Bezeichnung Shona eine negative Bedeutung und wurde auf alle Bevölkerungsgruppen übertragen, die nicht dem Ndebele-Stamm angehörten.

Eine Besonderheit der SHONA-Skulpturen liegt in der Eigenschaft des Materials, des Serpentinsteins (Serpentinit). Dieser wurde unter Einwirkung von Druck und Temperaturen in der Tiefe gebildet und im Laufe der Zeit durch tektonische Bewegungen an die Erdoberfläche gebracht. Auf dem afrikanischen Kontinent befinden sich markante Serpentinit-Vorkommen in Südafrika, Simbabwe und Äthiopien. Serpentinit tritt in unterschiedlichen Härtegraden und Farbnuancen auf, wobei Grüntöne besonders häufig vorkommen. Je nach Farbe werden die Steine unterschiedlich bezeichnet wie z. B. Springstone (schwarz), Opalit (leuchtend grün mit roten, orangen und blauen Flecken) oder Verdit (grün).

Das reichliche Vorkommen von Serpentinsteinen ermöglicht es vielen talentierten Menschen in Simbabwe, als Bildhauer tätig zu sein. Oft werden Themen aus Gesellschaft und Familie sowie aus Mythen und Legenden aufgegriffen. Häufig lassen sich die Künstler stark durch Form und Beschaffenheit des unbearbeiteten Steins inspirieren.

Die Shona-Kunst erhielt erste wichtige Anstöße, als sich in Simbabwe Tom Blumfield

Abb. 1: „Afrikanische Frau“ aus schwarzem Springstone.

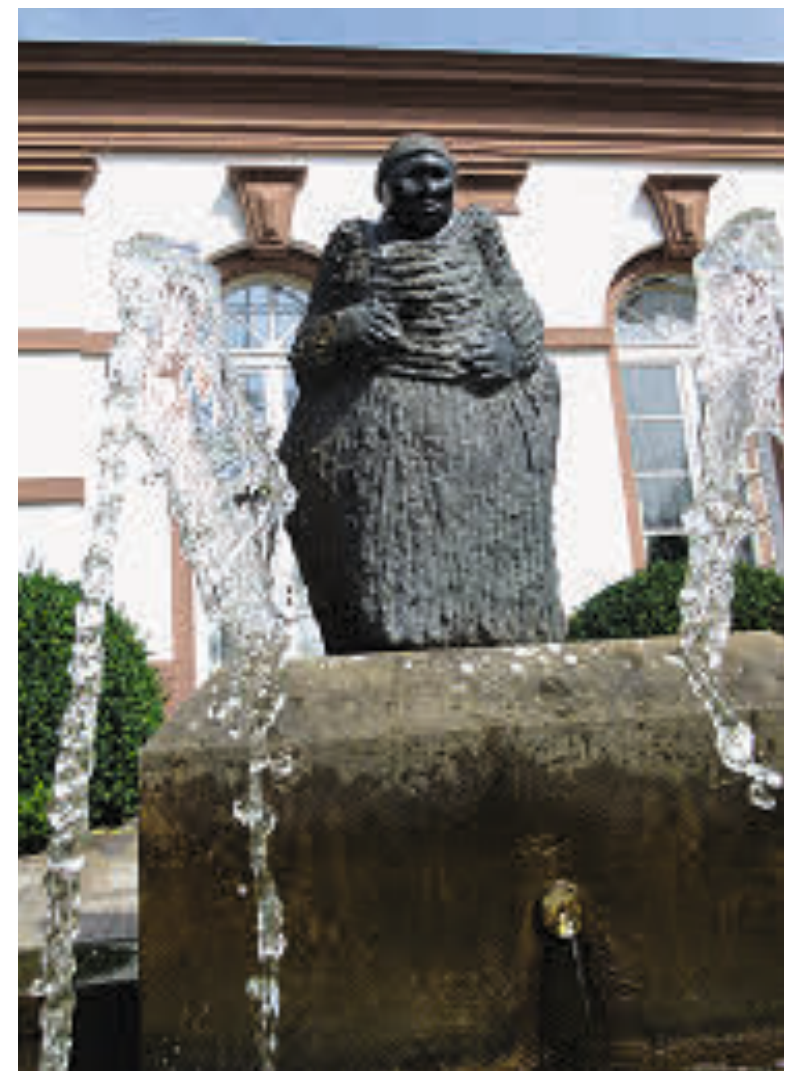

(geboren in Johannesburg, ehemaliger Bergmann aus dem Chromerzbergbau, später Farmer in Tengenenge) der Bildhauerei widmete. Als er merkte, dass seine schwarzen Farmarbeiter talentierter waren als er selbst, ermutigte er sie, die Steine künstlerisch zu bearbeiten und gründete eine Künstlerkolonie. Es entstanden dort zahlreiche Kunstwerke. Zwischen 1962 und 1972 konnte der Künstler Frank McEwen (Direktor der Nationalgalerie im damaligen Salisbury, 1907-1994) auf vielen internationalen Ausstellungen das Interesse für die afrikanischen Skulpturen wecken. Es gab Ausstellungen in New York und Paris, aber auch im Frankfurter Völkerkundemuseum und im Palmengarten.

Auch der Palmengarten besitzt mehrere SHONA-Kunstwerke. Die Frankfurterin Gertrud VolK, ehemaliges Mitglied der PalmengartenGesellschaft und dem Garten sehr zugetan, 


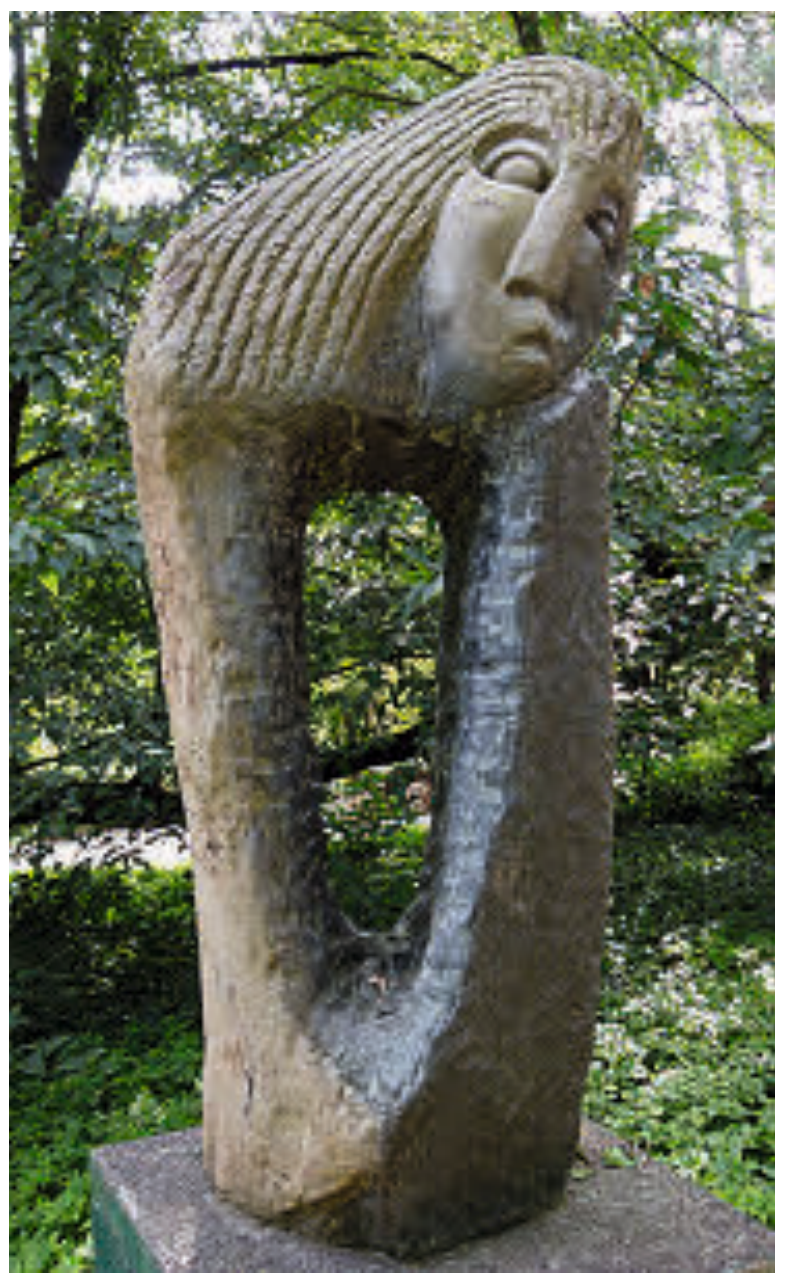

schenkte 1994 dem Palmengarten die $90 \mathrm{~cm}$ hohe Figur „Afrikanische Frau“. Sie wurde an der Westseite des Hauses Rosenbrunn neben einem kleinen plätschernden Brunnen aufgestellt. Die Bildhauerin Colleen Madamombe wurde 1964 in Simbabwe geboren. Sie ging nur fünf Jahre zur Schule, studierte aber drei Jahre lang Bildhauerei im Chapungu SkulpturenPark in Harare. Ihr großes Thema als Bildhauerin war die neu zu entwickelnde, selbstbewusste, stolze afrikanische Frau. Aus dem Jahr 2004 stammt ein Zitat von ihr, mit dem sie Frauen zur Bildhauerei ermutigen wollte: „Ich bitte Euch Frauen, ebenso als Bildhauer zu arbeiten wie die Männer. Ich weiß, das ist schwer, wenn ihr verheiratet seid. Die meisten Männer wollen nicht, dass ihre Frauen gleichberechtigte Bildhauer sind. Ich war verheiratet, und mein Mann war Bildhauer. Jetzt bin ich geschieden und sorge für meine sieben Kinder. Aber das ist in Ordnung." Ihre Kunstwerke fanden internationale

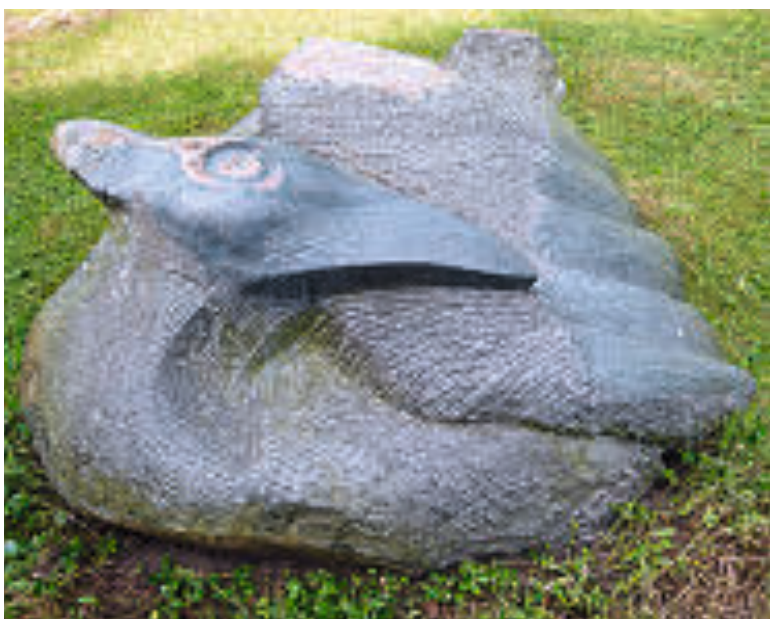

Beachtung, so auch auf der Expo 2000 in Hannover. Leider ist MaDAMOMBE viel zu früh am 31. Mai 2009 im Alter von nur 45 Jahren gestorben.

Zwischen dem Großen Weiher und der Liegewiese steht seit 1994 auf einem Sockel die $110 \mathrm{~cm}$ hohe Figur „Der bescheidene Beter“, der lustigerweise immer mal wieder falsch gelesen wird als „Der bescheidene Peter“. Die Figur wurde anlässlich der 1200-Jahr-Feier der Stadt Frankfurt von der Handwerkskammer Rhein-Main und der Iduna-Versicherung gestiftet. Geschaffen wurde die Skulptur vom Bildhauer Bernard TaKawira (geboren 1948 im Nyanga District). Der ehemalige Farmer und landwirtschaftliche Berater der Regierung gehörte zu den SHONA-Künstlern der ersten Generation. Seit 1977 arbeitete er in Vollzeit als Bildhauer. Er wurde durch McEwen gefördert und bekannt gemacht. TAKAWIRA starb 1997 in Harare.

Etwa in der Mitte des Palmengartens, abseits eines Weges auf einer Wiese am Bachlauf, liegt seit 1994 ein $65 \mathrm{~cm}$ hoher und über $100 \mathrm{~cm}$ breiter Stein-Vogel im Gras. Leider ist nicht bekannt, wer ihn erschaffen hat.

Abb. 2 (links): „Bescheidener Beter“.

Abb. 3 (rechts): Steinerner Vogel am Bachlauf. 